\title{
Ligand-induced symmetry breaking, size and morphology in colloidal lead sulfide QDs: from classic to thiourea precursors.
}

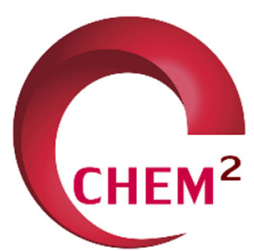

Federica Bertolotti a,b, Andrew H. Proppe c, Dmitry N. Dirin d,e, Mengxia Liu c, Oleksandr Voznyy c, Antonio Cervellino f, Simon J.L. Billinge g.h, Maksym V. Kovalenko d,e, Edward H. Sargent c, Norberto Masciocchi b, ${ }^{\text {i* }}$, Antonietta Guagliardi b, ${ }^{*}$

\section{Email: antonella.guagliardi@ic.cnr.it (iD)}

\author{
a Aarhus Institute of Advanced Studies (AIAS), Aarhus University, Høegh-Guldbergs Gade 6B, \\ 8000 Aarhus C, Denmark. \\ ${ }^{\mathrm{b}}$ Total Scattering Laboratory (To.Sca.Lab.), Via Valleggio 11, 22100 Como, Italy. \\ c The Edward S. Rogers Department of Electrical and Computer Engineering, University of Toronto, \\ 10 King's College Road, Toronto, Ontario M5S 3G4, Canada. \\ d Institute of Inorganic Chemistry, Department of Chemistry and Applied Biosciences, ETH Zürich, \\ Vladimir Prelog Weg 1, CH-8093 Zürich, Switzerland. \\ ${ }^{\mathrm{e}}$ Laboratory for Thin Films and Photovoltaics, Empa - Swiss Federal Laboratories for Materials Science \\ and Technology, CH-8600 Dübendorf, Switzerland. \\ f Swiss Light Source, Paul Scherrer Institut, 5232 Villigen, Switzerland \\ g Department of Applied Physics and Applied Mathematics, Columbia University, New York, New York \\ 10027, USA. \\ ${ }^{\mathrm{h}}$ Condensed Matter Physics and Materials Science Department, Brookhaven National Laboratory, Upton, \\ 11973 New York, USA. \\ ${ }^{i}$ Dipartimento di Scienza e Alta Tecnologia, University of Insubria, Via Valleggio 11, 22100 Como, Italy. \\ ${ }^{1}$ Istituto di Cristallografia, Consiglio Nazionale delle Ricerche, via Valleggio 11, 22100 Como, Italy.
}

\begin{abstract}
Colloidal lead chalcogenide quantum dots (CQDs) exhibit promising optoelectronic properties for applications in solar cell devices and as thermoelectrics. Herein, we report and discuss a ferroelectric structural distortion, at the picometer scale resolution, in PbS CQDs prepared using both classic and new synthetic pathways. The investigation was performed using synchrotron X-ray total scattering data and advanced methods of analysis that rely on a homo-core-shell model and evaluate the atomic arrangement, stoichiometry, size and morphology of nanocrystals. The CQDs show comparable size-dependent relative elongation, up to $0.7 \%$ of one body diagonal of the cubic rock-salt structure, which corresponds to a rhombohedral lattice deformation. The findings suggest a joint role for the oleate ligands (which induce surface tensile strain) and the $\mathrm{Pb}(\mathrm{II})$ lone pair as the driving forces of the deformation. Pb displacements along the [111] direction, which provoke a ferrolectric distortion related to the lattice change, fall in the $0.0-0.1 \AA$ range. Overall, the findings suggest the local nature of the metal off-centering, leading to different average displacements which depend on the synthetic conditions.
\end{abstract}

Keywords: Lead Chalcogenides, Quantum Dots, Nanocrystallography, X-ray Total Scattering, Debye Scattering Equation, Pair Distribution Function, Nanocrystal Morphology 


\section{Introduction}

The huge demand for (opto)electronic devices to be incorporated in a wide range of (small and large scale) applications, has acted as a massive incentive to scientific research for low-cost, solution-processed and easily scalable new semiconductors as alternatives to the silicon-based industry. Colloidal semiconductor nanocrystals, or quantum dots, represent valid potential substitutes: they provide tunable electronic properties achievable through precisely engineering their size, shape and surface chemistry and are easily integrated in devices as thin-films [1,2]. Lead chalcogenide ( $\mathrm{PbE}, \mathrm{E}=\mathrm{S}$, Se, Te) colloidal quantum dots (CQDs), exhibiting strong three-dimensional quantum confinement and ease of synthesis, are among the most investigated classes of semiconductor nanocrystals. Their possible use in optoelectronics, photodetectors, as promising thermoelectrics, electronic CMOS converters and, in particular, in solar cell devices, has engendered rapid advances in recent years [3-10]. Intense research has been addressed to optimize the synthesis in order to improve batch-to-batch reproducibility and reaction yield, while keeping or enhancing the photophysical performance. Hines and Scholes's synthetic method [11], based on the slow conversion of precursors, has been the most popular for years, with long-chain organic ligands (typically oleic acid) added on to stop the growth, passivate the nanocrystal surface and ensure their colloidal stability. In this method CQDs with very small sizes are obtained but also a batch-to-batch variability. Figure 1 schematically shows two of the classic pathways for PbS CQDs [using bis(trimethylsilyl) sulfide - (TMS) $)_{2} \mathrm{~S}$ - and lead acetate $\mathrm{Pb}(\mathrm{OAc})_{2}$ (TMS-1) or oxide $\mathrm{PbO}$ (TMS-2), as initial $\mathrm{Pb}$ precursors]. Based on the kinetic control of the precursors conversion reaction through a library of thioureas, Owen and co-workers [12,13] have recently proposed a new synthetic approach (also schematized in Figure 1, labeled as Thiourea) enabling the size of metal chalcogenide QDs to be tuned solely by the thiourea precursor reactivity and, at the same time, full conversion at a gram-scale yield. In a parallel way, relevant experimental and computational work has been dedicated over the years to investigate the surface-ligand interaction, due to the major role of the surface chemistry on the photophysical performance of QDs in the size range of interest (a few nm) [14,15]; however, a reliable description of the nanocrystal exposed facets (mainly pursued through high-resolution imaging techniques) remains a difficult task. In the framework of such evolving scenario, however, the effects of the severe downsizing and surface ligands on the internal nanocrystal structure have rarely been explored; these effects are expected to cause some deviations from the bulk structure; nevertheless, the undistorted atomic arrangement of the latter is commonly taken as the reference for the nanosize regime, although it may result in a rough approximation [16].

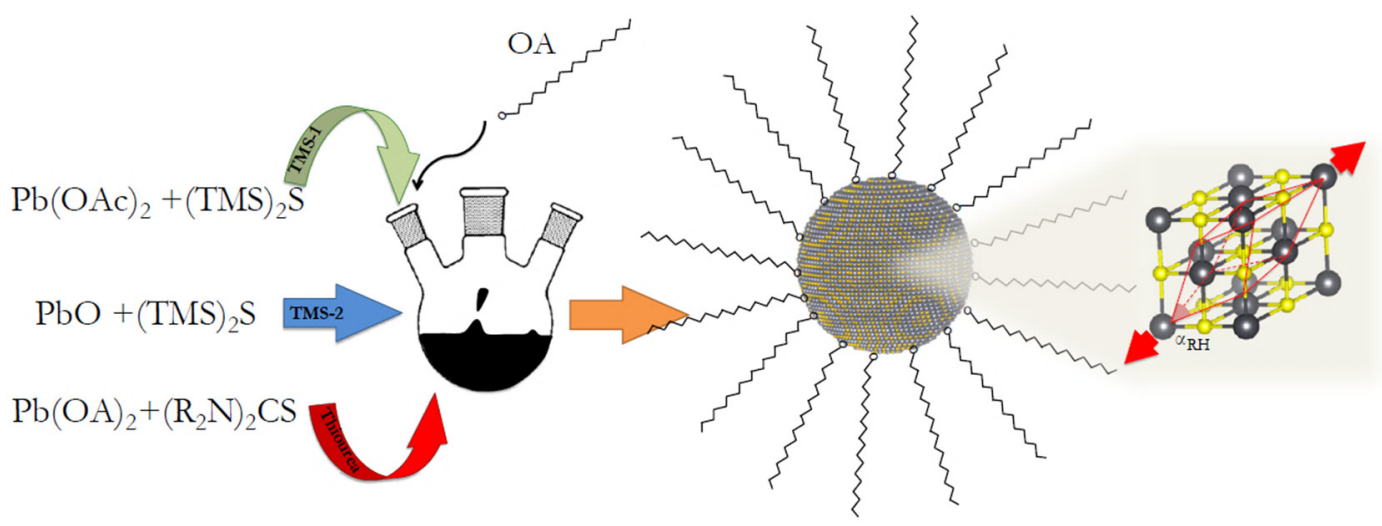

Figure 1. Schematic overview of precursors used in some classic (TMS-1 and TMS-2) and new (Thiourea-based) synthetic pathways of colloidal PbS quantum dots. For simplicity, OA ligands are drawn as saturated and fully stretched molecules with a single polar head.

The case of lead chalcogenide nanocrystals is emblematic. The highly symmetric rock-salt bulk structure is nearly ubiquitously reported for oleate-capped PbE CQDs; conversely, we have recently disclosed a size-dependent distortion from the cubic $(\mathrm{Fm}-3 \mathrm{~m})$ to the rhombohedral symmetry $(\mathrm{R} 3 \mathrm{~m})$ [17], detected jointly to the relative displacement of the $\mathrm{Pb}$ and E sublattices along one of the body diagonals of the cubic structure (red arrows in Figure 1, i.e. along the [111] axis, see Figure 2d for 
more details). Such displacement, one of the known cases of Pb off-centering, leads to three shorter and three longer $\mathrm{Pb}-\mathrm{E}$ bond distances, therefore determining a ferroelectric distortion in these materials, as much as observed in the $\alpha$-GeTe phase modification [18]. We found that this behavior in PbE CQDs is caused by two cooperative effects: the oleate-induced tensile stress (or, equivalently, the negative surface pressure, predicted to cause the change and expanding the cell volume by up to ca. $1.1 \%$ from the bulk upon downsizing [19]) and the stereochemical activity of the $\mathrm{Pb}$ (II) $6 s^{2}$ lone pair. The distortion was unveiled in 3-8 $\mathrm{nm}$ sized PbS and PbSe CQDs by advanced synchrotron $\mathrm{X}$-ray total scattering techniques and a complex homo-core-shell atomistic model of $\mathrm{PbE}$ nanocrystals combined to the Debye Scattering Equation (DSE) method. The distortion was further confirmed by DFT calculations in bulk PbS under the effect of a hydrostatic tensile stress [17].

The key-point of this finding is the insurgence of an electric dipole, due to the $\mathrm{Pb}$ off-centering, and its potential role in driving spontaneous chain-like aggregation and oriented attachment. Frequently reported in $\mathrm{PbE}$ QDs [20,21], these phenomena have been assigned to the presence of extended $\mathrm{Pb}$ - and E-terminated $\{111\}$ polar facets [20] despite that they conflict with the nearly unanimous consensus on the growth of nanocrystals with a Pb-terminated surface [22,23]. Thus, while the dipolar nature of the forces driving oriented aggregation is rather undisputed, its origin is still debated.

Once we detected the ferroelectric-type structural distortion in CQDs prepared by the TMS-1 synthesis (a slight modification of the Hines \& Scholes method, based on (TMS) ${ }_{2} \mathrm{~S}$ and $\mathrm{Pb}(\mathrm{OAc})_{2}$ precursors), we decided to explore any possible dependence of the distortion on the synthetic pathway. Herein, we report on the atomic- and nanometer -scale investigation of colloidal solutions prepared by following the modified and conventional Hines \& Scholes method (newly prepared TMS-1 and TMS-2, respectively, in Figure 1) and by approaching the synthesis in a completely different way, according to the thiourea-based method of Owen \& co-workers. The results point to a generalized effect in oleate-capped PbS CQDs; additional outcome of this investigation on the nanocrystals size and morphology, the exposed facets and related implications are also discussed.

\section{Results and Discussion}

PbS CQDs were synthesized according to TMS-1, TMS-2 and Thiourea syntheses. The different conditions and pathways between TMS- and thioureas -based approaches, which are mainly governed by thermodynamic and kinetic factors, respectively, may foster distinctive structural and morphological effects at the core and/or surface levels of PbS CQDs. In order to investigate and compare the three groups of QDs at atomic- and nanometer length scales, the as-prepared colloids, altogether having nominal sizes in the 3-7 nm range, were characterized by synchrotron Wide Angle X-ray Total Scattering (WAXTS) and advanced, unconventional methods of analysis, mainly in reciprocal space, through the DSE-based modeling. Complementary, real space Pair Distribution Function (PDF) analysis, Transmission Electron Microscopy and absorption spectroscopy were also used. Figure 2 gives an overview of synchrotron-based experiments and models. When collecting $\mathrm{X}$-ray diffraction data on particles of a few $\mathrm{nm}$ in size like QDs, size confinement, structural and compositional defects and surface relaxation result in broad peaks and a large amount of diffuse scattering. In this respect, we here highlight: $i$ ) the advantage of WAXTS experiments, compared to standard diffraction techniques, of being conceived to appropriately collect the entire (Bragg and diffuse) sample scattering; ii) the key strength of DSE and PDF modeling approaches (detailed in Materials and Methods) of encoding both Bragg and diffuse scattering in their pattern simulations, conversely to what is done by conventional Rietveld-based methods where only Bragg scattering is treated [24]; iii) the high brilliance and the extremely narrow wavelength dispersion $\left(\Delta \lambda \lambda=10^{-4} \div 10^{-5}\right.$, for $\left.\lambda \sim 0.5 \AA\right)$, intrinsic to the parallel synchrotron beam, providing a high signal/noise ratio and enabling the strict control of instrumental angular offsets, both concurring to the detection of distortions at a picometer-scale resolution, not easily feasible with laboratory measurements or other techniques [25].

The DSE method benefits from the description of nanocrystals at an atomistic level, which allows a number of relevant model parameters to be collectively considered within a unified model [26]. Such model includes the atomic arrangement, structural distortion and vacancies, nanoparticle size, morphology and surface relaxation [27-30]; the model is overall transferred into the pattern simulation in reciprocal space and can be optimized against the experimental WAXTS signal. As per the diffraction techniques, those parameters describe the ensemble of QDs in terms of distribution properties with high statistical significance, in contrast to microscopic techniques that probe local 
features on individual particles [31]. The relevant aspects of the DSE model applied to the collection of PbS CQDs are summarized hereafter.

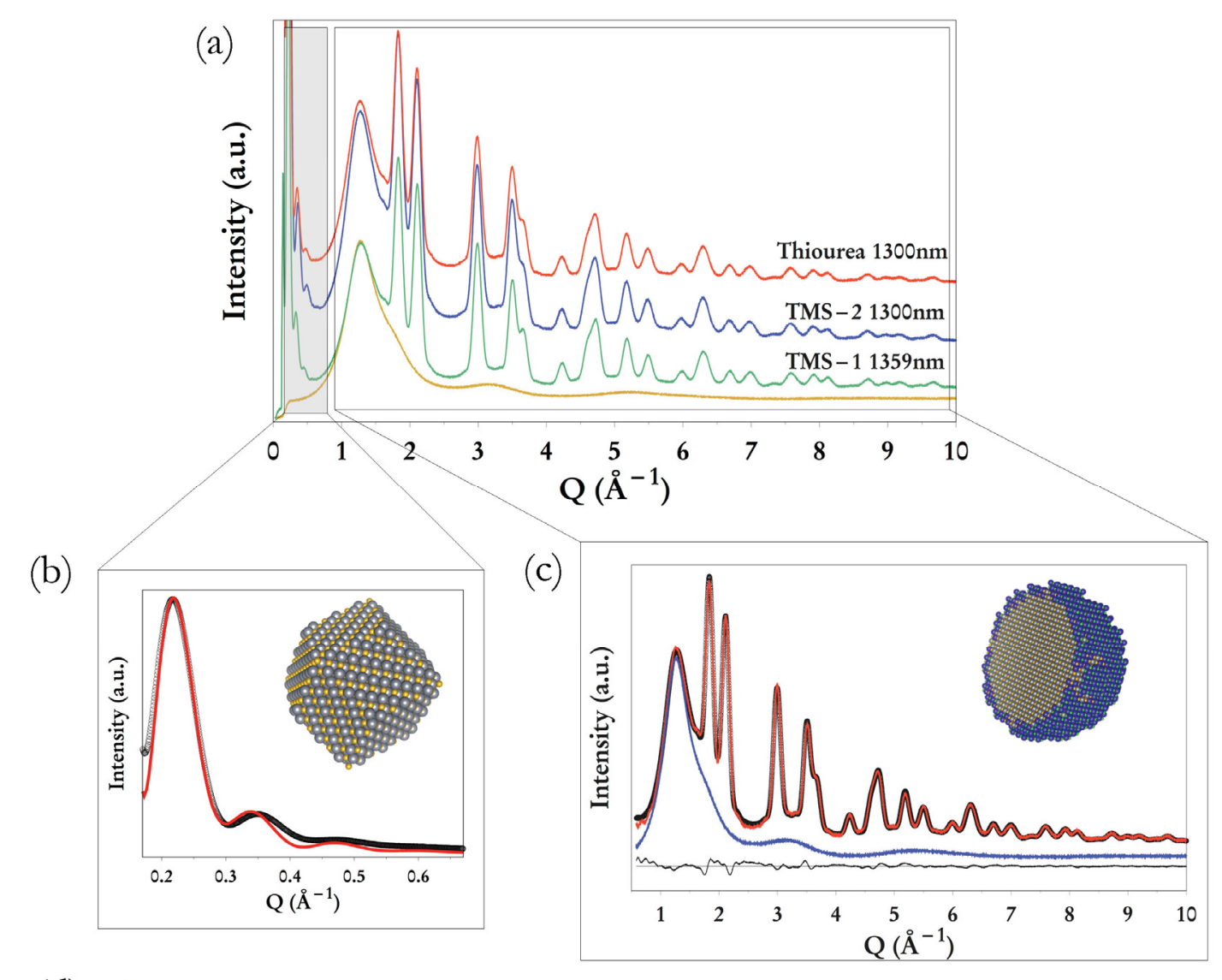

(d)
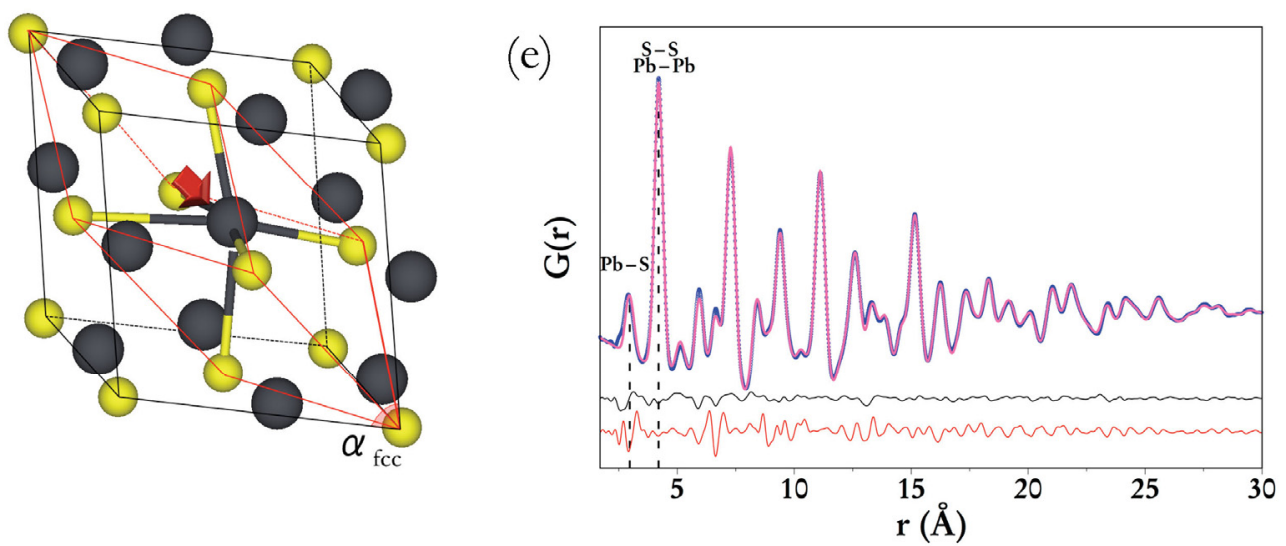

Figure 2. Synchrotron X-ray data collected on PbS CQDs in toluene (a) and DSE best models obtained for the thiourea-based synthesis, in the Porod small angle (b) and wide angle (c) regions; the two insets show the corresponding atomistic models used: the rhombic dodecahedral morphology, exposing only $\{110\}$ facets (b), and the homo-core-shell model (c) used for extracting atomic-scale information on the rhombohedral distortion, size and stoichiometry of QDs. (d) Ferroelectric distorted unit cell (fcc, black, and $\mathrm{RH}$, red) of $\mathrm{PbS}$ nanocrystals and relative displacement of Pb- (black) and S-sublattices (yellow). (e) Experimental (blue) and calculated (magenta) G(r) signals of the smallest (3.5 nm) TMS-1 sample.

The $\mathrm{G}(\mathrm{r})$ fit refers to the $R H$ structure; the difference plots are provided for the $R H$ (black) and $f c c$ (red) rock-salt models. 
Homo-core-shell spherical PbS nanocrystals (inset of Figure 2c) were built with adjustable core diameter, shell thickness and independent $\mathrm{Pb}: \mathrm{S}$ ratio for core and shell; the rock-salt structure was considered in the (equivalent) form of the primitive rhombohedral $(\mathrm{RH})$ unit cell (rather than the face centered cubic - fcc), with adjustable lattice parameter aRH and angle $\alpha \mathrm{RH}$, changing from $60^{\circ}$, (perfectly matching the undistorted fcc rock-salt structure) down to $58.50^{\circ}$ (for the RH-distorted lattices); the $\mathrm{Pb}$ displacement along the [111] direction was also refined. Figure 2a shows the WAXTS patterns collected on nearly equally-sized PbS CQDs, one for each synthesis, and Figures $\mathbf{2 b}$ and $\mathbf{2 c}$ the RH-distorted DSE best models fitting the Thiourea data, in the small and wide angle ranges, respectively; comparable fits are obtained for all the other samples (collected in the Supporting Information file, Figures S1 and S2). All the results provided by this model, in terms of QDs size, size-dependent $\mathrm{RH}$ distortion and Pb-shifts, surface strain, stoichiometry, and oleate coverage are discussed later on, in comparison to the results from other techniques. Figure $2 \mathbf{b}$ shows a very appealing extension of the DSE modeling to the low-angle WAXTS range $\left(0.15-0.65 \AA^{-1}\right)$, which corresponds to the Porod region typically measured by Small Angle $\mathrm{X}$-ray Scattering (SAXS); here the DSE method takes the advantage of using the atomic-scale structure as a reference for defining the nanocrystal exposed facets, and allows diverse morphologies to be investigated in highly monodisperse QDs [32]. Such morphological details are not detectable in the wide-angle range and enabled the rhombic dodecahedron (shown in the inset of Figure $\mathbf{2 b}$ ) to emerge as the preferential morphology in all as-synthesized QDs, regardless of the QDs size (see Figure S1). Such a finding points to the occurrence of $\{110\}$ non-polar facets only, exposed in freshly prepared colloids, with relevant implications on the driving forces contributing to $\mathrm{PbS}$ nanocrystals oriented attachment. As demonstrated elsewhere [17], and confirmed by these new syntheses, other feasible morphologies, exposing $\{111\}$ and $\{100\}$ polar facets do not provide likewise fits. Figure $2 \mathbf{d}$ depicts the ferroelectric distorted unit cell of $\mathrm{PbS}$ nanocrystals and the relative displacement of the $\mathrm{Pb}$ - and S-sublattices, which are discussed later. Figure 2e displays the best fit of the reduced G(r) function (addressing the probability of finding an atom at a distance $r$ away from another one) [33], provided by the complementary one-dimensional PDF method of analysis on the smallest TMS-1 sample. The refinement was carried out on both $f c c$ rock-salt and rhombohedral structures, upon relaxing (among the others) the lattice parameter $\left(a_{\mathrm{fcc}}\right.$ or $\left.a_{\mathrm{RH}}\right)$, the angle $\alpha_{\mathrm{RH}}(\mathrm{RH}$ model) and the Pb atom location (see details in Materials and Methods). PDF-analysis confirmed the $\mathrm{RH}$ distortion in all TMS-1 colloids (Figure S3).

The relevant results of this thorough analysis of PbS CQDs are summarized in Figures 3 and 4. The distortion from the highly symmetric fcc rock-salt structure to a rhombohedral one is here detected in all QDs, regardless of the synthetic method (see Tables S1-S4; statistical indicators are also reported for the $f c$ - and $\mathrm{RH}$-based modeling). The lattice distortion consists of a very tiny size-dependent elongation in the [111] direction, meaning that only one body diagonal (out of four) of the cubic arrangement is stretched. The largest relative elongation of ca. $0.7 \%$ found by DSE in the smallest QDs $(\sim 3 \mathrm{~nm})$ corresponds to $7.7 \mathrm{pm}$ and $1^{\circ}$ lowering of $\alpha_{\mathrm{RH}}$; at the largest size of $6.7 \mathrm{~nm}$, the corresponding values are close to the limit of detection (3.1 pm of elongation, $\alpha_{\mathrm{RH}}=59.6^{\circ}$ ). As Figure 3a displays, the higher the distortion, measured by the decrease of $\alpha_{\mathrm{RH}}$ (bottom), the larger the lattice expansion (top), given as the cubic-equivalent parameter $a_{f c}=\left(4 \mathrm{~V}_{\mathrm{RH}}\right)^{1 / 3}$, both being augmented by downsizing. PDF-derived values (green stars) confirm the same behaviour and are in line with those provided by DSE on the same TMS-1 CQDs.

In analogy to the size-dependent lattice expansion found in oxide nanoparticles and attributed to negative surface pressure effects [34], the dependence of $a_{f c c}$ on the QDs diameter $D$ of Figure 3a is described by the $a_{f c c}(D)=a_{0}(1-\Omega / D)$ equation, where the fitting parameters are $a_{0}$ (the bulk or cubic cell parameter for $D \rightarrow \infty$ ) and $\Omega=4 \gamma / 3 \mathrm{~B}$ ( $\gamma$ being the surface tension and B the bulk modulus). The black solid curve, fitting values from new and former samples, provides $\Omega=-0.014(2) \mathrm{nm}$ and $a_{0}=5.9255(23) \AA$ and nearly overlaps to that proposed in ref. [17] (red dashed curve, obtained by fitting the green squares only). Under the assumption that the bulk modulus value $(B=50 \mathrm{GPa})$ [35] can be used for PbS QDs, the negative surface tension $\gamma=-3.35 \mathrm{eV} \mathrm{nm}^{-2}$ is obtained, in line with the previous report [17]. In the TMS-1 CQDs, where such tensile strain was firstly observed, it was attributed to the long-chain organic ligands, the packing of which is not commensurate with the adsorption sites (on the $\{110\} \mathrm{PbS}$ facets). Herein, the detection in TMS-2 and Thiourea colloids of a lattice distortion identical than in TMS-1, also matching the same size-dependence, points to a systematic effect in oleate-capped PbS CQDs and to the major role of the oleic acid in inducing the distortion.

Related to the rhombohedral lattice deformation, the off-centering of the $\mathrm{Pb}$ ions in the [111] direction (further distorting the atomic arrangement in a ferroelectric manner) was detected by the DSE analysis in very small TMS-1 CQDs [17]. In this respect, surprising results are found in the present study, consisting in Pb-displacements that are spread in the 0.0-0.10 $\AA$ range (Figure 3b); while shifts are hardly detected at sizes $>5.0 \mathrm{~nm}$, where marginal $\mathrm{RH}$ distortions occur, it was unexpected measuring zero-value shifts at 
the smallest sizes, where nanocrystals display comparable $R H$ lattice distortion. Intriguingly, a regular patterning of the Pb-shifts emerges in the 3.1-3.6 nm CQDs, with the TMS-1 exhibiting the largest shifts (0.10-0.12 $\AA$, green points), the Thiourea CQDs the intermediate values (0.04-0.05 $\AA$, red points), whereas the shift vanishes in TMS-2 colloids (blue points). The off-centering of $\mathrm{Pb}$ ions in lead chalcogenides is indeed a highly questioned subject, intensely brought into play when the exceptional thermoelectric properties of these materials are concerned [36-40].

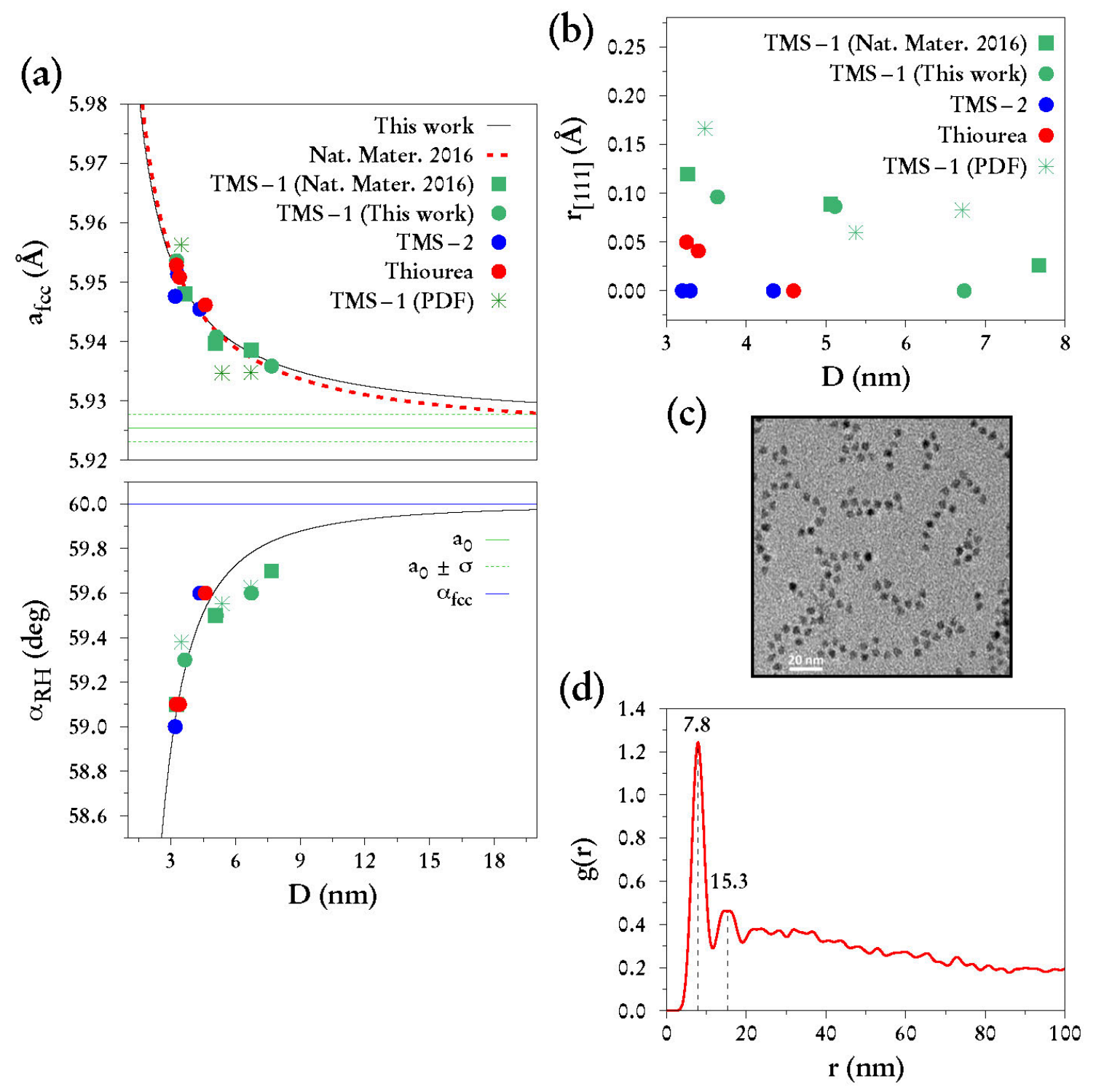

Figure 3. (a) Size-dependent RH-distortion in PbS CQDs; cubic-equivalent lattice parameter $a_{f c}$ (top) and angle $\alpha_{\mathrm{RH}}$ (bottom) vs QDs diameter, D. In the top part, the black solid curve fits the $a_{0}(1-\Omega / \mathrm{D})$ functional dependence; in the bottom part it is a guide for the eye. (b) Pb-displacements along the [111] direction vs QDs diameter, D; (c) TEM image displaying linear-chain aggregates forming in the $5.0 \mathrm{~nm}$

TMS-1 PbS CQDs; (d) radial distribution function $g(r)$ calculated from the TEM image in (c.

However, compared to these cases, despite of some analogies, the PbS CQDs here discussed are quite different. When investigating the role of the $\mathrm{Pb}$ off-centering in relation to the thermoelectric behavior, temperature-variable measurements (PDF, inelastic X-ray and neutron scattering) [39] and computational analyses (Maximum Entropy, Molecular Dynamics and Density Functional Theory) [36,37] are reported, indicating a tetragonal lattice modification and conflicting experimental evidences about the off-centering 
along the [100] direction. In 1D PDF analysis such conundrum is mainly attributed to anharmonic thermal vibration of the $\mathrm{Pb}$ ions, not accounted for in the modeling, making the discrimination between the two competing effects apparently impractical [38]. Similar side effects may be claimed in the DSE analysis here presented (anharmonicity is not considered); however, $\mathrm{Pb}$ off-centering is reported in bulk $\mathrm{PbE}$ even in the case in which anharmonicity is accounted for in the X-ray Rietveld model [36,37], therefore leaving the question still open. The PbS CQDs investigated in this work exhibit much stronger analogy with $\alpha-G e T e$, a narrow-gap semiconductor spontaneously transforming from the rock-salt to the polar rhombohedral modification below its Curie temperature $(\sim 675 \mathrm{~K})$ [41]. The $R H$ angular distortion $\left(1.65^{\circ}\right)$ is larger than in our QDs and the relative displacements of Ge and Te sublattices along the [111] direction are in the 0.05-0.40 A range, pointing to a variability of the reported values, similarly to our findings in PbS CQDs. Very stimulating, in this respect, are two recent papers $[42,43]$. The atomic-scale polar displacement maps were reconstructed on individual $8 \mathrm{~nm}$ nanocrystals of GeTe by combining high-resolution transmission electron microscopy and phase-recover algorithms. The position of $\mathrm{Ge}$ and Te columns were determined within an accuracy of few picometers and local polar displacements of ca. $0.31 \AA$ were found, showing, however, different degrees of linear correlations over the entire nanoparticle. This finding leads to an average polar displacement, in two distinct nanocrystals, of $0.29 \AA$ and $0.04 \AA$, for a $95 \%$ degree of correlation and a more complex pattern with mis-oriented polar domains, respectively. Therefore, single and multi-domain nanocrystals co-exist in the same sample. Additionally, T-dependent PDF analysis of bulk GeTe showed little or no change in the Ge-Te bond length upon increasing the temperature, proving that the structure remains locally distorted above the "phase-transition" to cubic metric; this finding points to an order-disorder rather than to a displacive transition in GeTe. Accordingly, the diverse $\mathrm{Pb}$-displacements observed in our PbS CQDs may result from the statistically averaging (over the entire ensemble of nanocrystals) of (nearly equal or even dissimilar) local polar displacements and with variable degree of linear correlations, apparently related to the synthetic pathway. However, in the absence of precise and accurate information on the local displacement in individual $\mathrm{PbS}$ nanocrystals (as in the case of Ge'Te), we can only postulate the existence of differently oriented polar domains, likely aligned along [111] or [-1-1-1] in our CQDs such that different Pb shifts and, at the same time, comparable lattice distortions are detected in average. The dependence of such degree of correlation on the synthetic method needs to be understood and additional investigations are required.

The TEM image of Figure 3c shows the spontaneous chain-like organization in $5.0 \mathrm{~nm}$ TMS-1 QDs, witnessing the presence of dipolar forces, which contradicts the centro-symmetry of the rock-salt crystal structure and, according to our morphological analysis (Figure 2b) also facets-driven dipolar interactions. Following the method proposed by Klokkenburg et. al. [44], we derived the dipole-dipole Pair Interaction energy for the QDs imaged in Figure 3c. For simplicity we adopted a single domain model for this calculation, attributing to each nanocrystal the corresponding average Pb displacement estimated for the entire sample. The radial distribution function $g(r)$ calculated from the image exhibits two peaks at $7.8 \mathrm{~nm}$ and $15.3 \mathrm{~nm}$ suggesting the predominant linear character of the aggregation. The effective inter-particle separation is $\sim 2.8 \mathrm{~nm}$ (the DSE-estimated QDs diameter is $5.0 \mathrm{~nm}$ ). Taking these values and the dipole moments calculated for a single domain nanoparticle as described in ref. [17], a pair interaction energy $\mathrm{V}=-10.6 \mathrm{k}_{\mathrm{B}} \mathrm{T}$ (or $\mathrm{V}=-17.9 \mathrm{k}_{\mathrm{B}} \mathrm{T}$, depending on which formula from ref. [44] is used) is obtained. Accordingly, when spontaneous alignment occurs in PbS CQDs, the dipolar structural distortion may be at work. Nevertheless, the additional role of the surface ligands cannot be ruled out. To complete the overview of the current analysis on the three synthetic approaches, we show in Figure 4 the comparison in terms of size and stoichiometry (their numerical values and other parameters are collected in the SI file, Table S1). Average number-based diameters and relative dispersion of TMS-1 PbS CQDs (Figure 4a) are in very good agreement with TEM-derived values and optical absorption spectra (Figures $4 \mathbf{b}$ and $\mathbf{4 c}$ ), calculated according to ref. [17]; size dispersions for Thiourea and TMS-2 syntheses (Figures 4d and 4e) parallel those of TMS-1. Regarding the refined structural parameters of the homo-core-shell model applied to the PbS CQDs investigated in this work (namely: atomic Debye-Waller factors, Pb vacancy in the core and $\mathrm{S}$ vacancy in the shell), values in line with those determined for TMS-1 subset in ref. [17], are found. Herein, Figure 4f shows comparable size-dependent $\mathrm{Pb}: \mathrm{S}$ ratios for the three syntheses. Taking into account the QD charge neutrality, ligands coverage of 2-3 oleates $\mathrm{nm}^{-2}$ are estimated (see SI file), regardless of the synthetic method. 


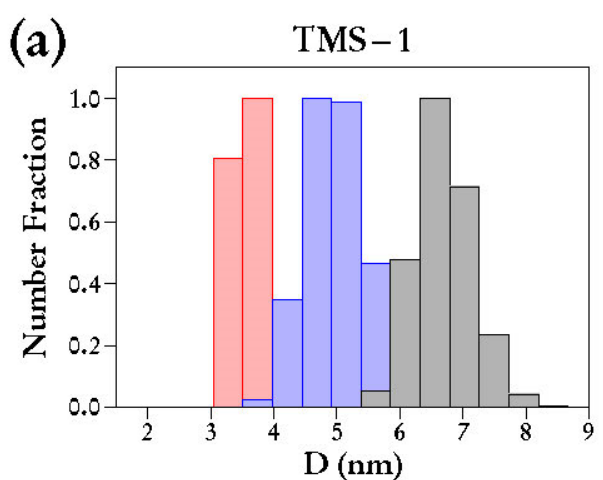

(b)

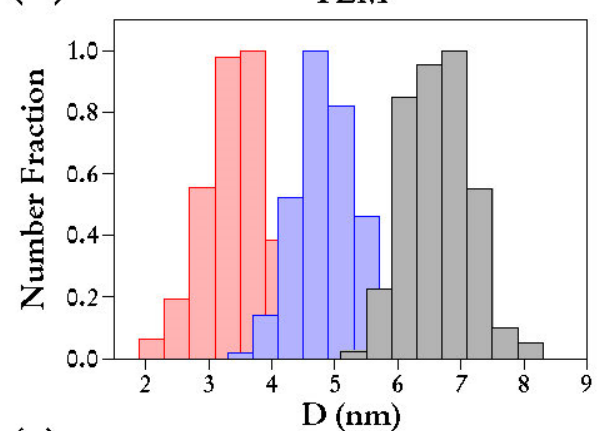

(c)

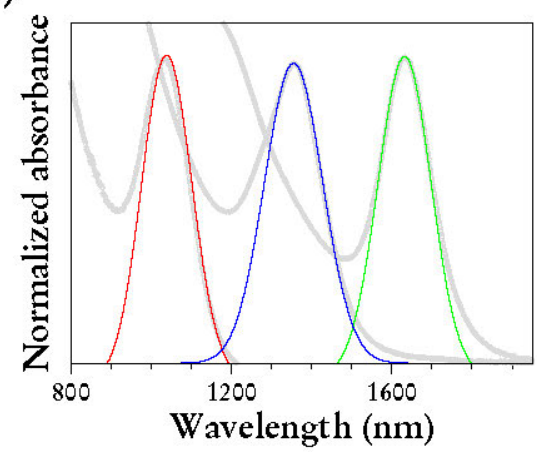

(d)

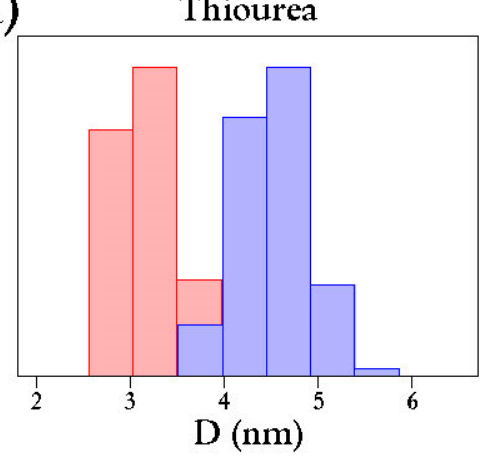

(e)

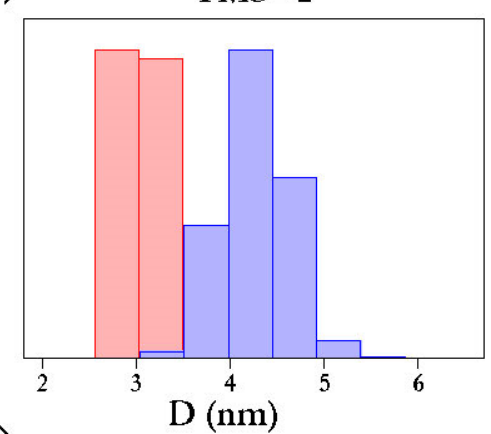

$(f)$

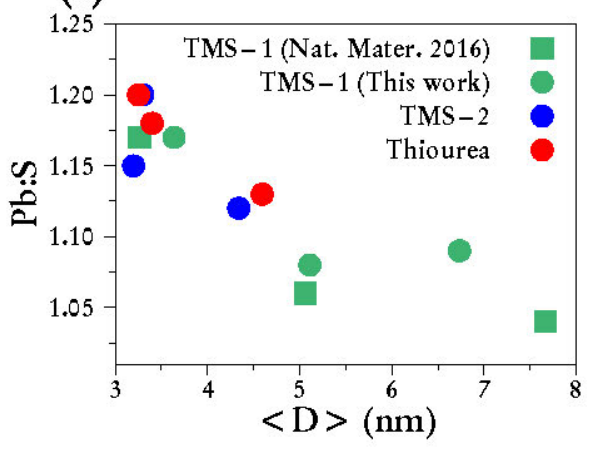

Figure 4. Comparison of the size analysis on TMS-1 CQDs by DSE (a), TEM (b) and absorption spectra (c); in (c) experimental (grey) and calculated (solid coloured lines) absorption spectra are overlaid. (d) DSE size analysis for the Thiourea and (e) TMS-2 set of samples. (f) Pb:S ratio determined by the DSE homo-core-shell model.

\section{Conclusions}

In this work we have investigated oleate-capped PbS CQDs synthesized according to different pathways, based on TMS and Thioureas as sulphur precursors. The analysis was carried out using advanced X-ray total scattering techniques and a unifying modelling of atomic-scale structure and nanocrystal size and morphology, relying on the Debye Scattering Equation method. DSE-based results are in line with those provided by other scattering, spectroscopic and imaging techniques. We demonstrated that a rhombohedral distortion of the $f c$ lattice occurs in all QDs, which exhibit a common size-dependence regardless of the synthetic method adopted, and point to the key role of the oleate ligands in inducing the distortion.

We also elucidate the nature of the $\mathrm{Pb}$ off-centering accompanying the lattice distortion, which consists in metal displacements along [111] or [-1-1-1] directions leading to average values spanning from 0.0 to 0.1 $\AA$ in the analyzed CQDs. The findings suggest locally distorted domains interconnected by a different degree of linear correlation that depends on the synthetic method. When not vanishing, the $\mathrm{Pb}$ 
displacement causes ferroelectric distortion and the formation of a permanent electric dipole moment in the nanocrystals. This finding may be of utmost importance for explaining the attachment of $\mathrm{PbE}$ nanocrystals forming linear aggregates, which are frequently imaged in lead chalcogenides nanocrystals and conflicts with the centrosymmetric nature of the rock-salt structure. Future work needs to be addressed in order to clarify the dependence of the different linear correlation of the $\mathrm{Pb}$ displacement on the synthetic method.

The influence of the synthesis on the morphology, size and size distributions of nanocrystals was also explored, in order to clarify the role of the precursors and synthetic mechanism in driving these fundamental features of $\mathrm{PbS}$ QDs and thus properly addressing their future implementations into functional devices. Sizes and size distributions, stoichiometry and ligand coverage appeared to be quite reproducible for the QDs here investigated, regardless of the recipe followed. The manuscript also shows the great potential of advanced total scattering techniques as an emerging tool to strengthen the material characterization at the nanoscale.

\section{Materials and Methods}

\section{Synthesis}

Materials. Lead (II) acetate trihydrate $\left(\mathrm{Pb}(\mathrm{OAc})_{2}\right.$, 99.999\%), bis(trimethylsilyl)sulfide ((TMS $\left.{ }_{2} \mathrm{~S}\right)$, 1-octadecene (ODE, 90\%), oleic acid (OA, 90\%), tributylphosphine (TBP, 97\%), selenium powder (99.99\%), aniline (>99.5\%), dodecylamine (>99\%), 4-(trifluoromethyl)phenyl isothiocyanate (97\%), phenyl isothiocyanate $(98 \%)$, hexane $(>95 \%)$, toluene $(\geq 99.9 \%)$, anhydrous tetrachloroethylene (TCE, $>99 \%)$, anhydrous methyl acetate $(99 \%)$, anhydrous octane $(>99 \%)$, and diethylene glycol dimethyl ether (diglyme, anhydrous, 99.5\%) were purchased from Aldrich. Absolute ethanol ( $\geq 99.8 \%)$ was purchased from Fluka. Tri- $n$-octylphosphine (TOP, 97\%) was purchased from Strem. Lead (II) oxide (PbO, 99.99\%) was purchased from Alfa Aesar.

TMS-1 synthesis. $\mathrm{Pb}(\mathrm{OAc})_{2}(1.5 \mathrm{~g}, 4 \mathrm{mmol})$ and varied amount of $\mathrm{OA}$ (2.8 mL for $3.2 \mathrm{CQDs}, 20 \mathrm{~mL}$ for $5.0 \mathrm{~nm}$ CQDs, $33 \mathrm{~mL}$ for $7.7 \mathrm{~nm}$ CQDs) were mixed in a three-neck flask and diluted to $50 \mathrm{~mL}$ by ODE. The mixture was degassed under vacuum at $120^{\circ} \mathrm{C}$ for 1 hour and heated to the injection temperature under argon flow. Injection temperatures were $103^{\circ} \mathrm{C}$ for the $3.2 \mathrm{~nm}$ sample and $145^{\circ} \mathrm{C}$ for 5.0 and $7.7 \mathrm{~nm}$ QDs. The heating mantle was removed and a solution of (TMS $)_{2} \mathrm{~S}(0.42 \mathrm{~mL}, 2 \mathrm{mmol})$ in $10 \mathrm{~mL}$ ODE (dried) was immediately injected into vigorously stirring lead oleate solution. After 3 min, the reaction mixture was cooled down to room temperature using a water bath. QDs were washed three times with a hexane/ethanol solvent/nonsolvent pair, redissolved in hexane or toluene and filtered through a $0.2 \mu \mathrm{m}$ PTFE filter. PbS CQDs of three nominally different sizes were prepared following this synthesis.

TMS-2 synthesis. Four samples of PbS CQDs of two nominally different sizes (950 and $1300 \mathrm{~nm}$ ) were prepared following previous published methods [11]. Specifically, for $950 \mathrm{~nm}$ CQDs, we used an injection temperature of $100^{\circ} \mathrm{C}$ and a $\mathrm{Pb}: \mathrm{S}$ ratio of 1.45 . For $1300 \mathrm{~nm}$ CQDs, we used an injection temperature of $130^{\circ} \mathrm{C}$ and $\mathrm{a} \mathrm{Pb}: \mathrm{S}$ ratio of 7.0 .

\section{Thiourea synthesis}

Synthesis of 4-(trifluoromethyl)phenyl-N'-phenylthiourea: $2.03 \mathrm{~g} \quad\left(\begin{array}{llll}\mathrm{mmol}\end{array}\right)$ of 4-trifluorophenylisothiocyanate and $0.93 \mathrm{~g}(10 \mathrm{mmol})$ of aniline were mixed in $10 \mathrm{~mL}$ of toluene and stirred for 2 hours until a white powder is precipitated. The product is isolated by evaporating the solvent and used without purification.

Synthesis of N-dodecyl-N'-phenylthiourea: $1.85 \mathrm{~g}(10 \mathrm{mmol})$ of $n$-dodecylamine and $1.34 \mathrm{~g}(10 \mathrm{mmol})$ of phenylisothiocyanate are mixed in $10 \mathrm{~mL}$ of toluene and stirred for 2 hours until a white powder is precipitated. The product is isolated by evaporating the solvent and used without purification. Three samples of PbS CQDs of two nominally different sizes (absoption peaks at 950 and $1300 \mathrm{~nm}$ ) were 
prepared following ref. [12]. Lead oleate $\left(\mathrm{Pb}(\text { oleate })_{2}\right)$ was synthesized from lead trifluoroacetate using the method of ref. [12] without any modification.

$950 \mathrm{~nm}$ CQDs: In a nitrogen glovebox, $440 \mathrm{mg}(0.572 \mathrm{mmol})$ of $\mathrm{Pb}$ (oleate) $)_{2}$ is dissolved in $15 \mathrm{~mL}$ of anhydrous octane, and $113 \mathrm{mg}$ of 4-(trifluoromethyl)phenyl-N'-phenylthiourea $(0.381 \mathrm{mmol})$ is dissolved in $0.5 \mathrm{~mL}$ of diglyme. Both solutions are sealed with septa, transferred to a Schlenk line under nitrogen pressure, and brought to $90^{\circ} \mathrm{C}$. After reaching a stable temperature, the thiourea solution is swiftly injected into the $\mathrm{Pb}$ (oleate) $)_{2}$ solution and allowed to react for 1 minute before the flask is removed from the heating mantle and allowed to cool to room temperature. The solvents are then removed under vacuum before the flask is transferred back into the nitrogen glovebox. $2 \mathrm{~mL}$ of toluene is added to dissolve the solids and then centrifuged at $7000 \mathrm{rpm}$ for 10 minutes to precipitate any large particles (aggregates). The solids that precipitate are discarded, and then $6 \mathrm{~mL}$ of methyl acetate is added to the supernatant before again centrifuging at $7000 \mathrm{rpm}$ for 10 minutes to precipitate the CQDs. The clear (or pale brown) supernatant is discarded and the CQDs are dissolved again in toluene. This process is repeated three times to wash the cQDs thoroughly.

$1300 \mathrm{~nm}$ CQDs: In a nitrogen glovebox, $440 \mathrm{mg}(0.572 \mathrm{mmol})$ of $\mathrm{Pb}$ (oleate $)_{2}$ is dissolved in $15 \mathrm{~mL}$ of anhydrous octane, and $127 \mathrm{mg}(0.381 \mathrm{mmol})$ of $\mathrm{N}$-dodecyl-N'-phenylthiourea is dissolved in $0.5 \mathrm{~mL}$ of diglyme. Both solutions are sealed with septa, transferred to a Schlenk line under nitrogen pressure, and brought to $120^{\circ} \mathrm{C}$. After reaching a stable temperature, the thiourea solution is swiftly injected into the $\mathrm{Pb}$ (oleate) $)_{2}$ solution and allowed to react for 10 minutes before the flask is removed from the heating mantle and allowed to cool to room temperature. The isolation and purification procedure are the same as described for the $950 \mathrm{~nm}$ CQDs.

\section{Experiments and Models}

Synchrotron X-ray total scattering measurements. High-resolution Wide-Angle X-ray Total Scattering (WAXTS) measurements were performed at the X04SA-MS4 beamline of the Swiss Light Source (Paul Sherrer Institute, Villigen, $\mathrm{CH}$ ) [25]. Colloidal suspensions of PbS QDs in toluene or hexane were loaded into borosilicate glass capillaries (Hilgenberg $\mathrm{GmbH} 0500, Q=0.8 \mathrm{~mm}$ ) and WAXTS data collected at beam energies of 22 and $25 \mathrm{KeV}$ in different experimental sessions, as follows: i) TMS-1. Data were collected on PbS CQDS in hexane at $25 \mathrm{KeV}(\lambda=0.49547592 \AA)$ and in toluene at $22 \mathrm{KeV},(\lambda=0.564162$ $\AA)$; ii) TMS- 2 and Thiourea. Data were collected in toluene, at $22 \mathrm{KeV}(\lambda=0.565483 \AA)$.

The wavelengths were accurately determined using a Silicon powder standard NIST (640d, $a=5.43123(8)$ $\AA$ at $\left.22.5^{\circ} \mathrm{C}\right)$. All datasets were collected in the $0.5^{\circ}-130^{\circ} 2 \theta$ range $\left(\mathrm{Q}=0.1-19 \AA^{-1}\right)$ using a single-photon counting Silicon microstrip detector (MYTHENII) [45]. Angle dependent intensity absorption corrections were applied to the raw data and to the air and capillary scattering contributions, independently measured on the empty container and the background; the latter were properly subtracted from the scattering of the sample [34]. Data of pure toluene and hexane were separately collected, in the same experimental conditions, and underwent the same data reduction procedure, to be used as part of the model (vide infra). High-energy data $(67 \mathrm{KeV}, \lambda=0.1827 \AA)$ for PDF analysis were collected on PbS TMS-1 CQDs in toluene filling kapton capillaries $(Q=1.0 \mathrm{~mm})$ at the 28-ID-2 XPD Beamline, National Synchrotron Light Source II (NSLS-II) of the Brookhaven National Laboratory (Upton, NY, US). The experimental data were measured using the Rapid Acquisition PDF mode and a Silicon-based 2D flat-panel detector (PerkinElmer), up to a momentum transfer $Q=23 \AA^{-1}[46]$. The $2 \mathrm{D}$ diffraction images were integrated using Fit2D [47] and the calibration parameters derived using a microcrystalline Ni standard. The toluene data, collected in the same experimental conditions, were subtracted from the $\mathrm{PbS}$ patterns in reciprocal space, before Fourier-transforming the data in real space.

DSE modeling of PbS CQDs. The DSE method provides the spherically averaged differential cross section of a randomly oriented ensemble of nanoparticles from the distribution of interatomic distances of each atom pairs in the particle, without any assumption of periodicity and order [48]. The DebUsSy Program Suite [26] is An open software project dedicated to characterize nanomaterials by the DSE method; its approach consists in generating atomistic models of nanoparticles in real space, encoding the information on interatomic distances in suitable databases, and using this information to calculate the DSE pattern in reciprocal space, where model parameters can be adjusted against the experimental data. For 
modelling the colloidal suspensions of PbS QDs in this work, similarly to what proposed in ref. [17], an atomistic homo-core-shell model was adopted and populations of spherical nanocrystals grown (at constant step $\Delta \mathrm{r}=0.47 \mathrm{~nm}$ ) with independent core diameter and shell thickness. A rhombohedral primitive unit cell with the angle $\alpha_{\mathrm{RH}}$ changing from $60^{\circ}$ (perfectly matching the undistorted face centered cubic lattice of the rock-salt structure) down to $58.50^{\circ}$ (for distorted lattices), in steps of $0.1^{\circ}$, was used as the building block and stored in different databases. In a subsequent step, the pattern model was calculated (in the $\mathrm{Q}=0.6-19 \AA^{-1}$ range) and several model parameters adjusted (using the Simplex algorithm [49]), for each $\alpha_{\mathrm{RH}}$ value: $i$ ) the average sizes and size distributions of each sample were refined according to a bivariate log-normal size distribution function (four adjustable parameters, including the core diameter, the shell thickness and their distributions); ii) the following structural parameters were refined: the rhombohedral lattice parameter $\left(a_{\mathrm{RH}}\right)$, the isotropic atomic thermal displacement $(a d p)$ factors for $\mathrm{Pb}_{\text {core }}$, $\mathrm{Pb}_{\text {shell }}, \mathrm{S}_{\text {core }}$ and $\mathrm{S}_{\text {shell, }}$, and site occupancy (sof) factors for $\mathrm{Pb}_{\text {core }}$ and $\mathrm{S}_{\text {shell. }}$. The rhombohedral angle of each sample was determined by performing different refinements at a step of $0.1^{\circ}$ as reported in [17], while the $\mathrm{Pb}$ coordinates along [111] were refined using the BOBYQA algorithm [50] starting from the best solution reached by the Simplex refinement.

Modeling the PbS morphology and faceting by the DSE method. The Porod region typically measured with a Small Angle X-ray Scattering experiment, was here collected as part of the WAXTS pattern $\left(0.15-0.65 \AA^{-1}\right)$ of PbS CQDs and modelled with the DSE method, using an atomic-scale approach, providing information on the QDs exposed facets [32]. Based on the crystal structure used for WAXTS modeling, we built $\mathrm{PbS}$ nanocrystals of rhombic dodecahedral morphology using $x_{2}, z$, coordinates satisfying the following geometrical conditions: $|x|+|y| \leq|r|,|y|+|z| \leq|r|,|x|+|z| \leq|r|$, with $r$ radius of the circumscribed sphere, and computed the corresponding Porod pattern using the DSE.

PDF analysis of PbS CQDs. The Total Scattering Function $S(Q)$ was obtained from the 1D X-ray pattern using the PDFgetX3 program [51]. The sine-Fourier transform is then applied to the reduced Total Scattering Structure Function, providing the experimental $G(r)$, which gives the probability of finding an atom at a distance $r$ away from another [33]. The model of $G(r)$ was computed using the program Diffpy-CMI [52], assuming an infinite crystal of rhombohedral symmetry and modeling its faster decay with $r$ by convoluting the $G(r)$ with a proper shape function, accounting for finite spherical nanocrystals. A log-normal size distribution function with two adjustable parameters (average size and its distribution) [53] was used to model the size dispersion of TMS-1 PbS CQDs. The following structural parameters were simultaneously refined: $a_{\mathrm{RH}}, \alpha_{\mathrm{RH}}$, adp's for $\mathrm{Pb}$ and $\mathrm{S}$, sof for $\mathrm{S}$ and the Pb off-centred shifts along [111].

Transmission Electron Microscopy. Transmission electron microscopy (TEM) was performed on a Tecnai F30 (FEI, FEG, $\mathrm{V}_{\text {acc }}=300 \mathrm{kV}$, SuperTwin lens, resolution ca. $2 \AA$ ).

Absorption Spectroscopy. Visible-near-infrared (VIS-NIR) absorption spectra for diluted colloidal solutions in TCE were collected using a Jasco V670 spectrometer.

Code availability. The DebUsSy Program Suite is freely available at http://debussy.sourceforge.net.

\section{Conflicts of interest}

The authors declare there are no conflict of interests.

\section{Acknowledgements}

F.B. acknowledges the European Union and the Aarhus Institute of Advanced Studies (Aarhus University) for the Marie Skłodowska-Curie AIAS-COFUND grant (EU-FP7 program, Grant Agreement No. 609033). S.J.L.B. was supported by NSF MRSEC/Columbia program (Center for Precision Assembly of Superstratic and Superatomic Solids, DMR-1420634). The technical staff of the X04SA-MS Beamline of the Swiss Light Source (PSI, Villigen, CH) is gratefully acknowledged. The measurements for PDF were 
conducted at NSLS II, a U.S. Department of Energy Office of Science User Facility operated by Brookhaven National Laboratory (Contract No. DE-SC0012704).

VII. References

[1] Kagan C. R., Lifshitz E., Sargent E. H., Talapin D. V., Science 2016, 353, aac5523-1-9.

[2] Kovalenko M. V., Manna L., Cabot A., Hens Z., Talapin D. V., Kagan C. R., Klimov X. V. I., Rogach A. L., Reiss P., Milliron D. J., Guyot-Sionnnest P., Konstantatos G., Parak W. J., Hyeon T., Korgel O. B. A., Murray C. B., Heiss W., ACS Nano 2015, 9, 1012-1057.

[3] Sun Z., Liu Z., Li J., Tai G. A., Lau S. P., Yan F., Adv. Mater. 2012, 24, 5878-5883.

[4] Chuang C.-H. M., Brown P. R., Bulović V., Bawendi M. G., Nat. Mater. 2014, 13, 796-801.

[5] Nugraha M. I., Hausermann R., Bisri S. Z., Matsui H., Sytnyk M., Heiss W., Takeya J., Loi M. A., Adv. Mater. 2015, 27, 2107-2112.

[6] Supran G. J., Song K. W., Hwang G. W., Correa R. E., Scherer J., Dauler E. A., Shirasaki Y., Bawendi M. G., Bulovic V., Adv. Mater. 2015, 27, 1437-1442.

[7] Lan X., Voznyy O., Garcia de Arquer F. P., Liu M., Xu J., Proppe A. H., Walters G., Fan F., Tan H., Liu M., Yang Z., Hoogland S., Sargent E. H., Nano Lett. 2016, 16, 4630-4634.

[8] Carey G. H., Abdelhady A. L., Ning Z., Thon S. M., Bakr O. M., Sargent E. H., Chem. Rev. 2015, 115, 12732-12763.

[9] Choi J.-H., Wang H., Oh S. J., Paik T., Jo P. S., Sung J., Ye X., Zhao T., Diroll B. T., Murray C. B., Kagan C. R., Science 2016, 352, 205-208.

[10] Ortega S., Ibanez M., Liu Y., Zhang Y., Kovalenko M. V., Cadavid D., Cabot A., Chem. Soc. Rev. 2017, 46, 3510-3528.

[11] Hines M. A., Scholes G. D., Adv. Funct. Mater., 2003, 1844-1849.

[12] Mark P. Hendricks, Campos M. P., Gregory T. Cleveland, Plante I. J.-L., Owen J. S., Science 2015, 348 , 1226-1230.

[13] Campos M. P., Hendricks M. P., Beecher A. N., Walravens W., Swain R. A., Cleveland G. T., Hens Z., Sfeir M. Y., Owen J. S., J. Am. Chem. Soc. 2017, 139, 2296-2305.

[14] Ip A. H., Thon S. M., Hoogland S., Voznyy O., Zhitomirsky D., Debnath R., Levina L., Rollny L. R., Carey G. H., Fischer A., Kemp K. W., Kramer I. J., Ning Z., Labelle A. J., Chou K. W., Amassian A., Sargent E. H., Nat. Nanotech. 2012, 7, 577-582.

[15] Dirin D. N., Dreyfuss S., Bodnarchuk M. I., Nedelcu G., Papagiorgis P., Itskos G., Kovalenko M. V., J. Am. Chem. Soc. 2014, 136, 6550-6553.

[16] Palosz B., Grzanka E., Gierlotka S., Stelmakh S., Z Kristallogr. 2010, 225, 588-598.

[17] Bertolotti F., Dirin D. N., Ibanez M., Krumeich F., Cervellino A., Frison R., Voznyy O., Sargent E. H., Kovalenko M. V., Guagliardi A., Masciocchi N., Nat. Mater. 2016, 15, 987-994.

[18] Rabe K. M., Joannopoulos J. D., Phys. Rev. B 1987, 36, 6631-6639.

[19] Zagorac D., Doll K., Schon J. C., Jansen M., Chem. Eu. J. 2012, 18, 10929-10936.

[20] Cho K.-S., Talapin D. V., Gaschler W., Murray C. B., J. Am. Chem. Soc. 2005, 127, 7140-7147.

[21] Evers W. H., Goris B., Bals S., Casavola M., de Graaf J., van Roij R., Dijkstra M., Vanmaekelbergh D., Nano Lett. 2013, 13, 2317-2323.

[22] Petkov V., Moreels I., Hens Z., Ren Y., Phys. Rev. B 2010, 81, 241304.

[23] Moreels I., Lambert K., Smeets D., Muynck D. D., Nollet T., Martins J. C., Vanhaecke F., Vantomme A., Delerue C., Allan G., Hens Z., ACS Nano 2009, 3, 3023-3030. 
[24] Cervellino A., Frison R., Masciocchi N., Guagliardi A. X-ray and Neutron Techniques for Nanomaterials Characterization. In: Kumar C.S.S.R. (ed). Springer-Verlag GmbH Germany: Berlin, Germany, 2016, 545-608.

[25] Willmott P. R., Meister D., Leake S. J., Lange M., Bergamaschi A., Boge M., Calvi M., Cancellieri C., Casati N., Cervellino A., Chen Q., David C., Flechsig U., Gozzo F., Henrich B., Jaggi-Spielmann S., Jakob B., Kalichava I., Karvinen P., Krempasky J., Ludeke A., Luscher R., Maag S., Quitmann C., Reinle-Schmitt M. L., Schmidt T., Schmitt B., Streun A., Vartiainen I., Vitins M., Wang X., Wullschleger R., J. Synchrotron Rad. 2013, 20, 667-682.

[26] Cervellino A., Frison R., Bertolotti F., Guagliardi A., J. Appl. Cryst. 2015, 48, 2026-2032.

[27] Bertolotti F., Moscheni D., Migliori A., Zacchini S., Cervellino A., Guagliardi A., Masciocchi N., Acta Crystallogr. A 2016, 72, 632-644.

[28] Bertolotti F., Protesescu L., Kovalenko M. V., Yakunin S., Cervellino A., Billinge S. J. L., Terban M. W., Pedersen J. S., Masciocchi N., Guagliardi A., ACS Nano 2017, 11, 3819-3831.

[29] Delgado-López J. M., Frison R., Cervellino A., Gómez-Morales J., Guagliardi A., Masciocchi N., $A d v$. Funct. Mater. 2014, 24, 1090-1099.

[30] Frison R., Cernuto G., Cervellino A., Zaharko O., Colonna G. M., Guagliardi A., Masciocchi N., Chem. Mater. 2013, 25, 4820-4827.

[31] Liu J., Olds D., Peng R., Yu L., Foo G. S., Qian S., Keum J., Guiton B. S., Wu Z., Page K., Chem. Mater. 2017, 29, 5591-5604.

[32] Gordon T. R., Diroll B. T., Paik T., Doan-Nguyen V. V. T., Gaulding E. A., Murray C. B., Chem. Mater. 2015, 27, 2502-2506.

[33] Egami T., Billinge S. J. L. (eds). Underneath the Bragg Peaks. Structural Analysis of Complex Materials: Kidlington, Oxford OX5 1GB, UK, 2003.

[34] Cervellino A., Frison R., Cernuto G., Guagliardi A., Masciocchi N., J. Appl. Cryst. 2014, 47, 1755-1761.

[35] Xu B., Wang Q., Tian Y., Sci. Rep. 2013, 3, 3068.

[36] Kastbjerg S., Bindzus N., Søndergaard M., Johnsen S., Lock N., Christensen M., Takata M., Spackman M. A., Brummerstedt Iversen B., Adv. Funct. Mater. 2013, 23, 5477-5483.

[37] Christensen S., Bindzus N., Sist M., Takata M., Iversen B. B., Phys. Chem. Chem. Phys. 2016, 18, 1587415883.

[38] Boris Sangiorgio, Emil S. Bozin, Christos D. Malliakas, Michael Fechner, Arkadiy Simonov, Mercouri G. Kanatzidis, Simon J. L. Billinge, Spaldin N. A., Weber T., arXiv:1709.02863v12017.

[39] Bozin E. S., Malliakas C. D., Souvatzis P., Proffen T., Spaldin N. A., Kanatzidis M. G., Billinge S. J., Science 2010, 330, 1660-1663.

[40] Jensen K. M. Ø., Božin E. S., Malliakas C. D., Stone M. B., Lumsden M. D., Kanatzidis M. G., Shapiro S. M., Billinge S. J. L., Phys. Rev. B 2012, 86, 08513.

[41] Chatterji T., Kumar C. M. N., Wdowik U. D., Phys. Rev. B 2015, 91, 054110.

[42] Polking M. J., Han M.-G., Yourdkhani A., Petkov V., Kisielowski C. F., Volkov V. V., Zhu Y., Caruntu G., Alivisatos A. P., Ramesh R., Nat. Mater. 2012, 11, 700-709.

[43] Matsunaga T., Fons P., Kolobov A. V., Tominaga J., Yamada N., Appl. Phys. Lett. 2011, $99,231907$.

[44] Klokkenburg M., Houtepen A. J., Koole R., Folter J. W. J. d., Erné B. H., Faassen E. v., Vanmaekelbergh D., Nano Lett. 2007, 7, 2931-2936.

[45] Bergamaschi A., Cervellino A., Dinapoli R., Gozzo F., Henrich B., Johnson I., Kraft P., Mozzanica A., Schmitt B., Shi X., J. Synchrotron Rad. 2010, 17, 653-668.

[46] Chupas P. J., Qiu X., Hanson J. C., Lee P. L., Greya C. P., Billinge S. J. L., J. Appl. Cryst. 2003, 36, 1342-1347.

[47] Hammersley A. P., ESRF Internal Report, ESRF97HA02T 1997. 
[48] Debye P., Ann. Phys. 1915, 351, 809-823.

[49] Nelder J. A., Mead R., Comput. J. 1965, 27, 308-313.

[50] Powell M. J. D. The BOBYQA algorithm for bound constrained optimization without derivatives: Cambridge: University of Cambridge; 2009.

[51] Juhás P., Davis T., Farrow C. L., Billinge S. J. L., J. Appl. Cryst. 2013, 46, 560-566.

[52] Juhás P., Farrow C. L., Yang X., Knox K. R., Billinge S. J., Acta Crystallogr. A 2015, 71, 562-568.

[53] Gamez-Mendoza L., Terban M. W., Billinge S. J. L., Martinez-Inesta M., J. Appl. Cryst. 2017, 50, 741-748.

Received: 07 December 2017

Accepted: 23 January 2018

Published online: 05 February 2018

ORCID ID for authors

Federica Bertolotti: 0000-0002-6001-9040

Andrew H. Proppe: 0000-0003-3860-9949

Dmitry N. Dirin: 0000-0002-5187-4555

Mengxia Liu: 0000-0002-1676-705X

Oleksandr Voznyy: 0000-0002-8656-5074

Antonio Cervellino: 0000-0002-9086-2717

Simon J.L. Billinge: 0000-0002-9734-4998

Maksym V. Kovalenko: 0000-0002-6396-8938

Edward H. Sargent: 0000-0003-0396-6495

Norberto Masciocchi: 0000-0001-9921-2350

Antonietta Guagliardi: 0000-0001-6390-2114

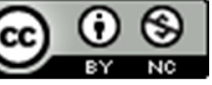

This article is licensed under a Creative Commons Attribution-NonCommercial 4.0 International License, which permits use, sharing, adaptation, distribution and reproduction in any medium or format, as long as it is non-commercial, you give appropriate credit to the original author(s) and the source, provide a link to the Creative Commons license, and indicate if changes were made. The images or other third-party material in this article are included in the article's Creative Commons license, unless indicated otherwise in a credit line to the material. If material is not included in the article's Creative Commons license and your intended use is not permitted by statutory regulation or exceeds the permitted use, you will need to obtain permission directly from the copyright holder. To view a copy of this license, visit http://creativecommons.org/licenses/by/4.0/. 